\title{
Vapour pressure isotope effect on evaporation from pure organic phases - A PIMD approach
}

\author{
Luis Vasquez and Agnieszka Dybala-Defratyka a) \\ Institute of Applied Radiation Chemistry, Lodz University of Technology, Żeromskiego 116, Eódź 90-924, \\ Poland
}

(Dated: 4 August 2020)

Often in order to understand physical and chemical processes taking place among several phases fractionation of naturally abundant isotopes is monitored. Its measurement can be accompanied by theoretical determination to provide a more insightful interpretation of observed phenomena. Predictions are challenging due to the complexity of the effects involved in fractionation such as solvent effects and non-covalent interactions governing the behaviour of the system which results in the necessity of using large models of those systems. This is sometimes a bottleneck and limits the theoretical description to only a few methods.

In this work vapour pressure isotope effects on evaporation from various organic solvents (ethanol, bromobenzene, dibromomethane, and trichloromethane) in the pure phase are estimated by combining force field or self-consistent charge density-functional tight-binding (SCC-DFTB) atomistic simulations with path integral principle. Furthermore, the recently developed Suzuki-Chin path integral is tested. In general, isotope effects are predicted qualitatively for most of the cases, however, the distinction between position-specific isotope effects observed for ethanol was only reproduced by SCC-DFTB, which indicates the importance of using non-harmonic bond approximations.

Energy decomposition analysis performed using the symmetry-adapted perturbation theory (SAPT) revealed sometimes quite substantial differences in interaction energy depending on whether the studied system was treated classically or quantum mechanically. Those observed differences might be the source of different magnitudes of isotope effects predicted using these two different levels of theory which is of special importance for the systems governed by non-covalent interactions.

Keywords: path integral molecular dynamics, nuclear quantum effects, vapour pressure isotope effects, isotope fractionation, organic solvents, SCC-DFTB, interaction energy, non-covalent interactions

\section{INTRODUCTION}

Understanding that vapour pressures for different polyatomic compounds do not depend categorically on molecular weight, is considered one of the major breakthroughs in chemistry 8 . Therefore eventually, solvent effects, structural configuration, and non-covalent interactions were characterised as vital phenomena to take into account while investigating volatilization process.

One of the most successful methods to study organic solvents volatilisation has been isotope effect measurement. Interpretation of measured isotope effects on vaporisation is system dependent ${ }^{9} 11$, and can be explained using two main accepted approaches so far: i) the Craig-Gordon model (CGM) ${ }^{\sqrt{9 \mid 12}}$ together with its modifications ${ }^{13}[17$, for which the total isotope effect consists of the equilibrium vapour-pressure enrichment and transport resistance, and ii) the physical chemistry model, where isotope effects mainly arise from non-covalent interactions $7 / 8|11| 18,22$.

Altogether, measured isotope effects are classified into two groups depending on which phase is enriched with the heavier isotopologue, normal if it is the liquid phase or inverse if it is vapour. Principally, for the last two decades, CGM has been systematically used for organic

\footnotetext{
a) Electronic mail: agnieszka.dybala-defratyka@p.lodz.pl
}

solvents $\frac{16 \mid 23,25}{2}$, to a large extent due to technological advances such as gas chromatography isotopic ratio mass spectrometry (GC-IRMS), gas chro matography multiple ion collectors inductively coupled plasma mass spectrometry (GC-MCICPMS), multicollector-inductively coupled plasma mass spectrometer (MC-ICPMS), and isotope ratio monitoring by nuclear magnetic resonance $(\mathrm{NMR})^{26}$ among the most popular.

On the other hand, the physical chemical approach has been adopted for a longer time $8|11| 19,22|27| 29$. Likely because the transport resistance term is considered a negligible correction therefore it does not affect the relative overwhelming equilibrium vapour pressure enrichment $10[30$. Nevertheless, recent studies have shown that, isotope transport resistance takes relevance in certain environmental sceneries ${ }^{16 / 31 / 32}$ up till the point to counteract the equilibrium enrichment 16 . Furthermore, Julien et al! 24 have investigated and demonstrated that diffusion must be taken into account as the CGM indicates. A short description of driving forces upon fractionation process under different environments is provided in Table 1 of Ref 33.

Calculating isotope effects for a known isotopic exchange reaction

$$
\mathrm{X}_{(\text {liq })}+\mathrm{X}_{(\text {gas })}^{\prime} \stackrel{\Delta \mathrm{G}}{\rightleftharpoons} \mathrm{X}_{(\text {liq })}^{\prime}+\mathrm{X}_{(\text {gas })}
$$

where $\mathrm{X}$ and $\mathrm{X}$ ' are the light natural abundant and heavy isotopologues, respectively, is principally performed using 
the approximation given by Bigeleisen and Mayer ${ }^{3}$. It is vital to have in mind that isotope effects are solely an aftereffect of molecule quantum mechanical fluctuations, thence, the theoretical study of such, must be performed by the means of proper computational quantum chemistry tools. For example, Yanase and Oi performed simulations using electronic structure calculations of molecular orbitals3435, where vapour pressure isotope effects (VPIE) of some halogenated systems ${ }^{20127}$ were predicted successfully using a modified version of Bigeleisen and Mayer's approximation ${ }^{3435}$. Their findings highlighted that Bigeleisen and Mayer's approximation provides unreliable predictions of deuterium isotopic substitution ${ }^{3134}$.

Hence it is obvious that proper and/or ultimate ways for theoretical prediction of isotope effect, surrogate to Bigeleisen and Mayer's approach are impelling needed, especially when isotope effects modelling can provide information regarding structure, intermolecular interactions $34 \sqrt[35]{35}$ and forces dominating the process.

Feynman's imaginary time path integral (PI) formulation of quantum mechanics ${ }^{36 \sqrt[38]{38}}$ has been used successfully in order to estimate isotope effects by including nuclear quantum effects $(\mathrm{NQE})^{39} \sqrt{43}$ from molecular dynamics (MD) simulations, thanks to the quantumclassic isomorphism ${ }^{44}$. Path integral molecular dynamics (PIMD) offers an alternative and reliable way to estimate VPIE, by directly quantising kinetic energy differences between isotopes $\frac{45 / 46}{}$, and relating that difference to the Helmholtz's free energy for a given isotopic exchange reaction 47 .

In our previous study we performed ${ }^{13} \mathrm{C}$ and ${ }^{81} \mathrm{Br}$ VPIE predictions for bromobenzene ( $\mathrm{BB}-\mathrm{C}_{6} \mathrm{H}_{5} \mathrm{Br}$ ), and dibromomethane (DBM - $\left.\mathrm{CH}_{2} \mathrm{Br}_{2}\right)^{48}$ evaporation using PIMD. That study revealed that although PIMD can be used for predicting isotope effects on evaporation from pure organic phase consisting of aforementioned compounds, estimators with faster convergence, accurate reproduction of potential energy surface, and better sampling techniques for its computation needed to be used. With that in mind, in the current work we recompute ${ }^{13} \mathrm{C}$ and ${ }^{81} \mathrm{Br}$ VPIEs for BB and DBM systems. Moreover, we compare the predicted magnitudes with experimentally determined isotope effects on pure phase vaporisation at standard conditions of other halogenated organic compound (chloroform) $)^{29}$ highly abundant in water and/or great concern as environmental pollutant, and an alcohol (ethanol) ${ }^{2429}$ which exhibits differences in its positions specific carbon isotope effect. Structural characteristics of the models comprising liquid phase of studied systems are analysed in order to identify relevant solvent - solvent interactions, and asses the overall reliability in faithfully reproducing the potential surface energy. Finally, we demonstrate the capabilities of the self-consistent charge density-functional tight binding (SCC-DFTB) PIMD for some of the studied systems.

\section{METHODS}

MD simulations were performed for bromobenzene (BB $\mathrm{C}_{6} \mathrm{H}_{5} \mathrm{Br}$ ), dibromomethane (DBM - $\mathrm{CH}_{2} \mathrm{Br}_{2}$ ), chloroform $\left(\mathrm{TCM}-\mathrm{CHCl}_{3}\right)$, and ethanol $\left(\mathrm{ETH}-\mathrm{C}_{2} \mathrm{H}_{5} \mathrm{OH}\right)$ - see Table I- using the open-source software LAMMPS (largescale atomic/molecular massively parallel simulator) ${ }^{49}$, developed by the Sandia National Laboratory. Optimized potentials for liquid simulations (OPLS) force field parameters ${ }^{50151}$ were taken from the LigParGen web server ${ }^{52}$. System size for pure phase simulations comprised 90 molecules of each compound, distributed uniformly in a cubic box of $15 \AA$ to which periodic boundary conditions were applied. Using step-size equal to $0.5 \mathrm{fs}$, time integration (Nosé-Hoover style ${ }^{53}$ ) on nonHamiltonian equations of motion ${ }^{54}$ were computed over a total of $1 \cdot 10^{7}$ steps at $297.15 \mathrm{~K}$ and $0.1 \mathrm{MPa}$ in order to equilibrate the systems to their respective experimental density (NPT), and generate sufficient data for posterior statistical analysis. For all molecular dynamics simulation the reversible reference system propagator algorithm (rRESPA) ${ }^{55}$ multi-timescale integrator was used.

Isotope fractionation factor at temperature $T$, by means of Helmholtz's free energy can be expressed as $\alpha=e^{\beta \Delta G}$, where $\Delta G$ represents the free energy consequent to isotope substitution, and $\beta=1 / k_{B} T$. In the direct scaledcoordinates estimator by Cheng and Ceriotti, $\frac{42}{4}$ that isotope fractionation factor can be given as the ratio of phases ensemble average in the exchange reaction (Eq. (1p), thus, for instance, ${ }^{13} \mathrm{C}$ isotope effect on ethanol evaporation using the aforementioned estimator would be as follows

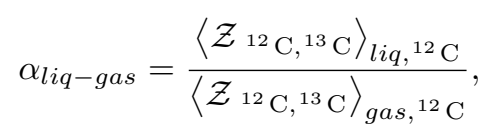

where

$$
\begin{aligned}
& \mathcal{Z}^{12 \mathrm{C},{ }^{13} \mathrm{C}} \equiv \exp \left[-\frac{\beta}{P} \sum_{k=1}^{P} V\left(q_{1}^{\prime(k)}, \ldots, q_{N}^{(k)}\right)-\right. \\
& \left.V\left(q_{1}^{(k)}, \ldots, q_{N}^{(k)}\right)\right] \text {, }
\end{aligned}
$$

the summed elements are differences between potential energies $(V)$ values of given system at k-th imaginarytime slice with exclusion of the particle being isotopically substituted and the same potential energy values for all involved particles. For consistency with the experimental data, predicted isotope effects are given in terms of enrichment factor $\varepsilon$, which is calculated as

$$
\varepsilon=\frac{1}{n}\left(\frac{1}{E I E}-1\right) \cdot 1000
$$

where $n$ represents the number of atoms of the same element in a molecule (Sec. II), and EIE is the equilibrium isotope effect reflecting equilibrium between liquid and vapour phases. 
TABLE I. Systems used in this study

\begin{tabular}{l}
\hline Name \\
\hline Bromobenzene (BB) \\
Dibromomethane (DBM) \\
Chloroform (TCM) \\
Ethanol (ETH)
\end{tabular}

Calculations were performed for the systems ongoing the isotopic exchange reaction in Eq. (1). PIMD simulations were performed using the path-integral interface i-PI ${ }^{56}$. Two substitutions, namely ${ }^{13} \mathrm{C}$ and ${ }^{81} \mathrm{Br}$ were taken into account. In cases where more than one atomic position had to be taken into account (e.g. two bromine atoms in DBM), fractionation factor is given as the geometric average of position specific isotope effect, unless otherwise stated. In order to estimate isotope fractionation, the scaled-coordinates estimator (SC) was used 40142 . In our previous work ${ }^{48}$, we determined empirically that at least 28 imaginary time-slices, as for BB, were needed in order to converge the estimator. Such finding is within the range of the rule of thumb, $P=2 \beta \hbar \omega_{\max }$. Thus, having limitations regarding computational cost in mind, simulation time needed for system convergence during PIMD, and the size of system in this study, PIMD simulations were performed using 32, 64, and 128 imaginary time-slices. PIMD simulations were carried out using the canonical ensemble NVT at $297.15 \mathrm{~K}$, with a time step of 0.25 fs using the "global" Path Integral Langevin thermostat (PILE-G) for an efficient sampling 57 .

\section{RESULTS AND DISCUSSION}

\section{A. Structural analysis of studied liquids}

In order to confirm that the potential energy surface of the studied compounds are being properly reproduced by the force field, first we analysed the structural properties of the compounds and compared them to available experimental data. Computed average densities for all studied systems are presented in Table III. Results indicate that in general, used integrator tends to slightly overestimate density values. While using MD simulations to equilibrate the system under study, it is commonly expected that deviations from experimental densities do not surpass $4 \% \underline{58}$, and indeed this is the case for all studied compounds, except TCM, where the discrepancy from the experimental density is $7 \%$. However, taking into account the density standard deviation in the performed MD simulation, the estimated error is as low as $4.4 \%$.

TABLE II. Experimental and predicted values of density at 297.15 K @ 0.1 MPa.

\begin{tabular}{lcc}
\hline & \multicolumn{2}{c}{ Density $\left[\mathrm{g} \mathrm{cm}^{-3}\right]$} \\
\cline { 2 - 3 } Compound & $\mathrm{MD}$ & Exp. \\
\hline Bromobenzene & 1.517 & $1.495^{\mathrm{a}}$ \\
Chloroform & 1.596 & $1.479^{\mathrm{a}}$ \\
Ethanol & 0.782 & $0.785^{\mathrm{b}}$ \\
Dibromomethane & 2.472 & $2.477^{\mathrm{c}}$ \\
\hline Ref. [59. & & \\
bRef. 60. & & \\
CRef. 61. & &
\end{tabular}

In order to assess the quality of MD simulations, comparative structural analysis between resulted trajectories and corresponding experimental data was performed. Analyses for BB are shown in Fig. 1

We computed radial distribution functions (RDFs). In the case of RDFs, we notice that before the second peak in the bromine-carbon RDF (Fig. 1] bottom-right plot) a small peak is forming. It is even clearer when this work results are compared to our previous work 48 . Bertagnolli et al. detected that within the second shell (3.7 $\AA)$ there was another minor peak, formed more likely due to interactions between free electrons of the benzene ring and a bromine atom on top of the ring plane and van der Waals interaction at larger distances ${ }^{62}$. Such interactions must have been practically absent in the models resulted from our previous simulations using the general AMBER force field parameters, however, OPLS FF counts for them. Bromine-bromine RDF (Fig. 1, bottom-left plot) reflects that, contrary to our previous work (4.2 $\AA$ ), OPLS FF 

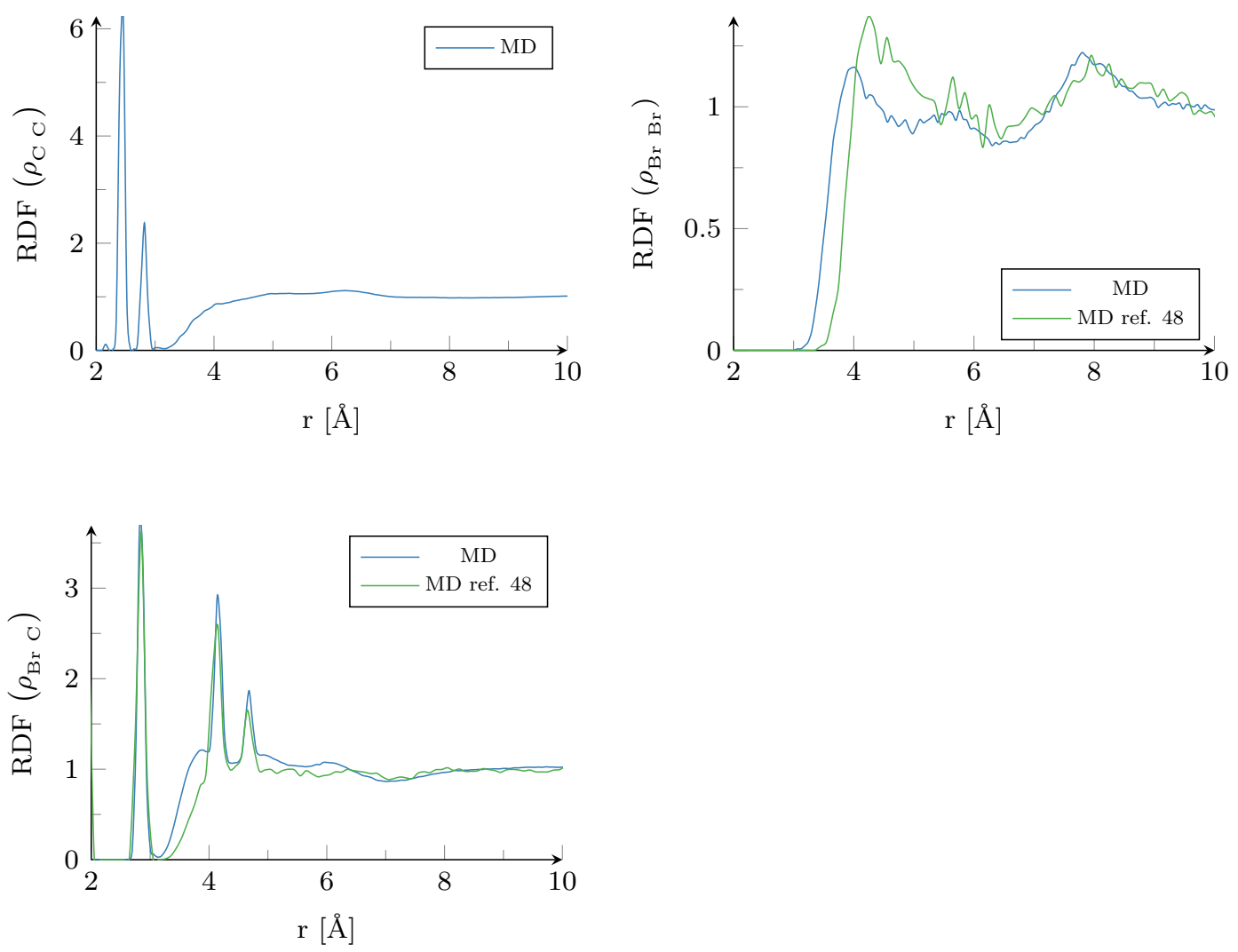

FIG. 1. computed carbon-carbon radial distribution function (top-left), bromine-bromine RDF (top-right), and bromine-carbon RDF (bottom-left) from MD simulations for bromobenzene.

predicts closer bromine-bromine interactions (3.8 $\AA$ ).

Structure of liquid TCM has been recently studied by Pothoczki et al $\underline{63}$, while MD simulations and $a b$ initio calculations for the same system have been performed by Yin et al. ${ }^{64}$. Figure 2 presents plots of RDFs based on the simulations using OPLS FF performed for TCM.

In comparison to the experimental data, RDF computed for the carbon atom around other carbon atoms, overestimates the magnitude of the first peak by around $30 \%$, and in general RDFs are shifted to larger distances. The largest disparity is seen in RDF computed for the hydrogen atoms (Fig.2 bottom-left plot), where simulations do not capture the first shell interactions at $2.8 \AA$, the second shell at $5 \AA$ is overestimated by almost $70 \%$ with a significant shift to larger distances, and the third shell is missing. These observations indicate that the first shell is not reproduced by the used force field, while the second one is substantially overestimated. This behaviour rises from the short range $\mathrm{H}-\mathrm{H}$ and $\mathrm{C}-\mathrm{H}$ interactions that are part of the repulsive potential region ${ }^{64}$. These interactions were not included in the fitting procedure of OPLS FF that relies more on fitting thermochemical properties 50 .

Computed RDFs for ethanol do not reflect meaningful differences between molecular dynamics simulations and experimental data ${ }^{6566}$, (Fig. 3).

Interestingly, $\mathrm{RDF}$ for $\mathrm{C}_{1}-\mathrm{C}_{2}$ (see Table $\mathrm{I}$ for atoms naming) reveals that the first shell extends from around 4 to $5.5 \AA$, an unusual feature as compared to other system in this study, where peaks tend to be sharp. Coordination number (for a central atom, the number of atoms by which it is surrounded) was calculated for $\mathrm{C}_{1}-\mathrm{C}_{2}$ in order to see the implications of its peculiar RDF. The result indicates that on average at $4 \AA$ only one carbon atom can be found, while at $5.5 \AA$ the number of atoms exceeds 7. This result does not allow to neglect the $\mathrm{C}_{1}-\mathrm{C}_{2}$ interactions, as they could play an essential role in the behaviour of liquid ethanol. Coordination number (ncoord) for O- $\mathrm{H}_{\mathrm{O}}$, and $\mathrm{O}-\mathrm{O}$ pairs to the first minimum of the respective RDF, were equally computed and compared to Gereben \& Pusztai $\frac{67]}{6}$ calculations. O- $\mathrm{H}_{\mathrm{O}}$, and $\mathrm{O}-\mathrm{O}$ ncoord are identical to Gereben's values, namely 1 and 2 respectively. Given result complements the selection of the OPLS force field model.

Based on our analyses, it is clear that to a certain degree, the interatomic potential energy surface of the studied systems is well reproduced by the OPLS force field. Perhaps, the best example is that of ETH, as the force field mimics relatively well pair distributions and structure factor.

In the case of DBM, although experimental density was successfully reproduced, no experimental data regarding its structure was found, therefore the trajectories for 

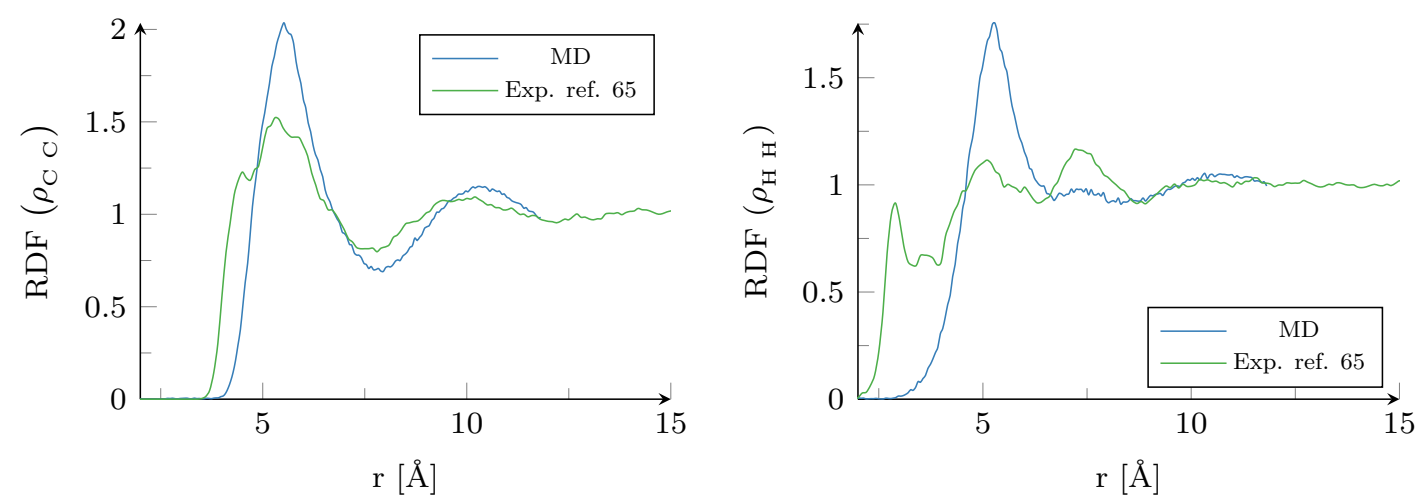

FIG. 2. Computed carbon-carbon RDF (top-left), and hydrogen-hydrogen RDF (top-right) from MD simulations for TCM.
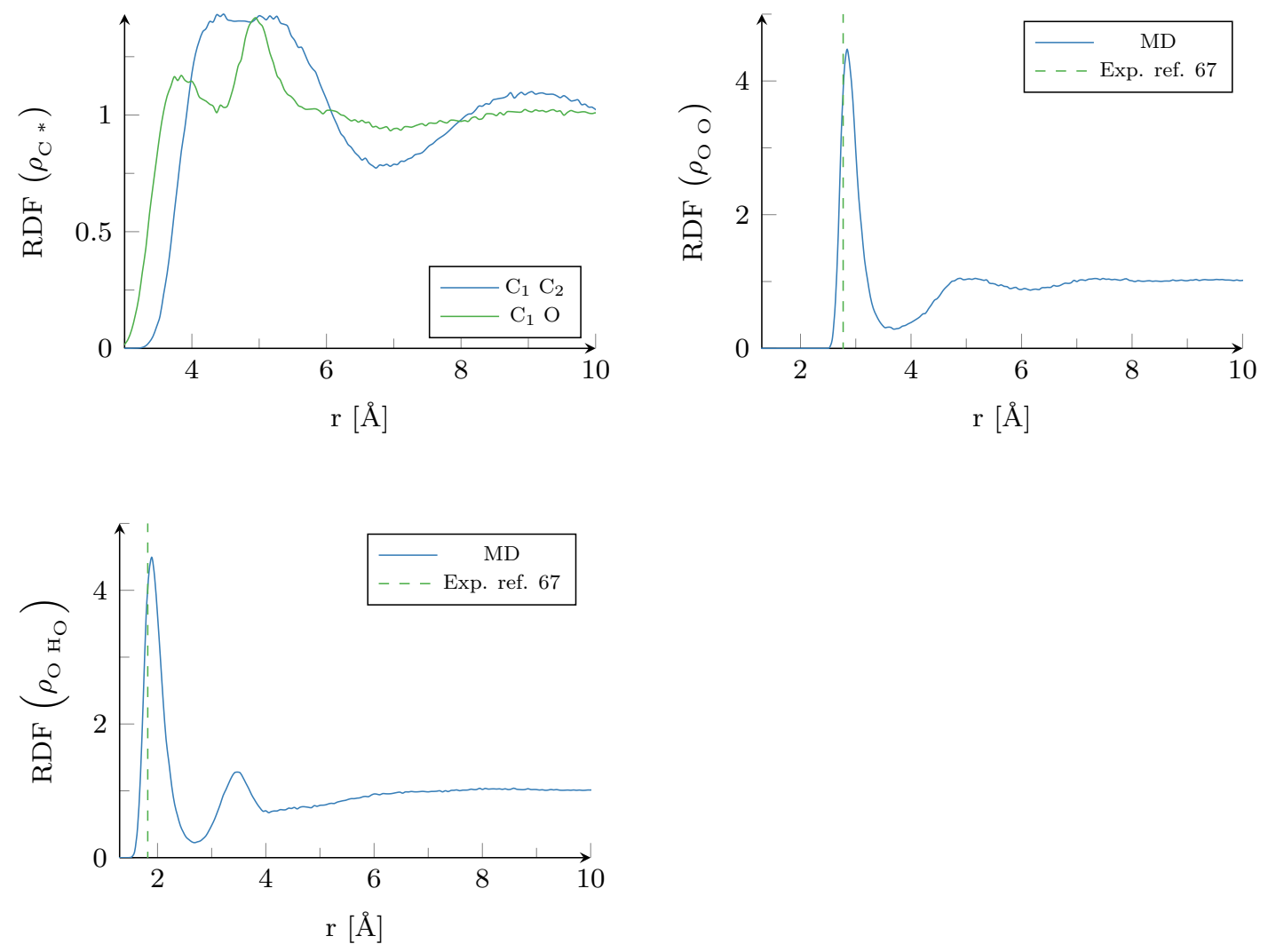

FIG. 3. Computed carbon-carbon and carbon-oxygen RDF (top-left), oxygen-oxygen RDF (top-right), and oxygen-hydrogen(hydroxylic hydrogen) RDF (bottom-left) from MD simulations for ethanol.

DBM simulations were analysed but left as supplementary material (see Fig. S1. in Supplementary Material).

\section{B. Predicted VPIEs}

Fractionation factors for vaporization of the studied compounds (BB, TCM, DBM, and ETH) obtained using the SC estimator are given in Table [III The observed magnitudes, except for TCM and DBM for which pre- dicted carbon isotope effects are normal in contrast to the experimental inverse effect, are qualitatively in good agreement with experimental data. All measured VPIEs used in this work come from evaporation studies at room temperature ${ }^{24 / 48}$. All computed isotopic substitutions in this work using PIMD always lead to normal carbon VPIE of the same magnitude.

The reason for this behaviour should be sought in the nature of isotope effect. Since they are quantum phenomena, they are caused by the change in zero-point energy 
TABLE III. VPIEs $(\varepsilon \%)$ on evaporation for bromobenzene, dibromomethane, chloroform, and ethanol using PIMD and OPLS force field.

\begin{tabular}{|c|c|c|c|c|c|}
\hline \multirow[b]{2}{*}{ Compound } & \multirow[b]{2}{*}{ isotope } & \multicolumn{3}{|c|}{ Time slices } & \multirow[b]{2}{*}{ Exp. } \\
\hline & & 32 & 64 & 128 & \\
\hline $\mathrm{BB}$ & ${ }^{81} \mathrm{Br}$ & $-1.04 \pm 1.47$ & $-1.04 \pm 1.47$ & $-1.06 \pm 1.49$ & $-0.9 \pm 0.2^{\mathrm{a}}$ \\
\hline TCM & ${ }^{13} \mathrm{C}$ & $-1.00 \pm 0.59$ & $-0.99 \pm 0.58$ & $-0.99 \pm 0.58$ & $1.2^{\mathrm{c}}$ \\
\hline DBM & ${ }^{13} \mathrm{C}$ & $-1.00 \pm 0.71$ & $-0.99 \pm 0.70$ & $-1.01 \pm 0.71$ & $0.6 \pm 0.2^{\mathrm{a}}$ \\
\hline DBM & ${ }^{81} \mathrm{Br}$ & $-1.08 \pm 1.75$ & $-1.08 \pm 1.72$ & $-1.03 \pm 1.67$ & $-0.8 \pm 0.1^{\mathrm{a}}$ \\
\hline
\end{tabular}

${ }^{a}$ Ref. 48

Carbon isotope effect for carbon bonded to $\mathrm{Br}\left(\mathrm{C}_{1}\right.$ in $\mathrm{BB}$ from Table I $)$

CRef. 24 by passive evaporation.

due to molecular vibrations of isotope exchange and this can be determined only using electronic structure methods. However, even accurate quantum methods do not account for solute-solvent interactions which are crucial in liquids. That is why in order to include intermolecular interactions present in the solvent, empirical potentials are often used as they are relatively cheap to perform. One natural computational solution that would take into account quantum behaviour of the solute and allowed for proper sampling of the environment with its fluctuations effect on the solute would be using a combined quantum mechanical and molecular mechanical (QM/MM) potential. However, despite numerous, successful applications to condensed phase processes (mostly reactions), when it comes to its use along with the PI formalism to predict isotope effects, the treatment of the QM region is often limited to using semi-empirical potentials which are fast but not always accurate enough, in particular when substantially small equilibrium isotope effects were of interest $\frac{68}{}$. Better, but of course not always feasible, solution could be treating all molecules present in the system at the equally accurate - $a b$ initio level. However, since, ab initio molecular dynamics approach limits the application to small systems only we decided to test one semi-empirical method first, namely the self-consistent charge density-functional tight binding (SCC-DFTB)

It must be mentioned that although in principle Morse potential force fields can potentially be used, a SCCDFTB is more appealing. Not only SCC-DFTB provides a quantum mechanical characterisation of the studied systems, but it is also fundamentally parameter free. With those two characteristics, it is expected that a proper description of halogen atoms and position specific isotope effect of carbon in ethanol molecule can be achieved.

A common issue seen in the predicted VPIEs is the large standard deviation that systems present, especially for halogen atoms regardless the imaginary time slices number used. Longer simulations for both gas and liquid phase did not lead to the reduction of standard deviation, therefore a different approach must be sought. In the i-PI interface, an inexpensive way to improve convergence without increasing the number of imaginary slices is the
Suzuki-Chin PIMD ${ }^{70}$ 73, which uses a finite-difference integrator instead of the traditional Trotter decomposition (conventional PIMD) for integrating high order terms that arise from commutators of the kinetic and potential energy. Therefore, we tested it to predict ${ }^{13} \mathrm{C}_{1}$ VPIE on ethanol evaporation. The test revealed that in general, aside from a insignificant decrease of $0.005-0.01 \varepsilon \%$ in the standard error, there was no significant improvement. Under those circumstances, it is clear that 32 imaginary slices are more than enough to converge the system.

One restriction in the validity of using FF for the simulation of quantum effects, concretely for VPIEs predictions, it is that most of them do not take into account anharmonicity contributions. It is specially important for liquid phase interactions and hydrogen-bonded systems such as water and ethanol. Therefore, in order to include the aforementioned contributions, the selfconsistent charge density-functional tight binding (SCCDFTB) PIMD simulations were performed using i-PI jointly with the DBTB+ ${ }^{74}$ software.

Third order density functional tight binding (DFTB3) parameters ${ }^{75176}$ including corrections for non-covalent interactions, dispersion and hydrogen bonds, were used (DFTB3-D3H5) ${ }^{77}[80$ for the computation of forces in the different imaginary time slices. Moreover, with the intention of improving and speeding convergence, the so-called path integral generalized Langevin equation thermostat (PIGLET) ${ }^{57 / 81}$ was employed. Regardless the acceleration provided by the PIGLET scheme, as atoms are being evaluated quantum-mechanically, system sizes were reduced to 15 or 21 molecules based on the coordination numbers from MD analysis, and the number of imaginary time slices scaled down to 8 and 16. Systems were minimised in order to be posteriorly equilibrated for 10 ps employing the NPT ensemble.

In order to perform $S C C$-DFTB PIMD simulations, a range of time steps from 1 to 0.5 fs was tested with the aim to determine a time step value that would allow to collect data faster without compromising the stability of simulations. It was established that $0.5 \mathrm{fs}$ would be the best choice. To ensure that enough statistical data would be collected, 250 ps for gas phase and 50 ps of simulation 
for liquid phase were performed.

Within the studied compounds, VPIEs using $S C C$ DFTB PIMD could have only been estimated for BB and ETH, while DBM, and TCM presented serious issues in the self-consistent charge convergence. Our explanation for these problems points to halogen atoms as the source of those difficulties. There are more than one either chlorine or bromine atom in the molecules of TCM or DBM, respectively. $\mathrm{BB}$ which also has a halogen atom in its structure presented some complications too, but to a lesser extent. In contrast, ethanol did not reflect any SCC convergence problems.

$S C C$-DFTB PIMD VPIEs results (Table IV) differ vastly from the conventional PIMD data (Table III), in particular for ethanol. Not only predicted ${ }^{81} \mathrm{Br}$ VPIE for bromobenzene is closer to the experimental data than VPIE predicted using PIMD in force field, but also predictions are achieved with a smaller number of imaginary slices. In contrast to the predicted ${ }^{13} \mathrm{C}$ VPIE using force field potential, $S C C$-DFTB PIMD allowed for capturing differences between position specific isotope effect, namely ${ }^{13} \mathrm{C}_{1}$ and ${ }^{13} \mathrm{C}_{2}$. This highlights the importance of using quantum mechanical approaches, instead of harmonic-like force field potentials.

Generally speaking, we observed that standard error deviations are substantially reduced. For instance, in the case of simulations for ethanol with 8 time imaginary slices, its standard deviation (SD) amounts to less than half of that for PIMD using force field and 32 slices. On the other hand, standard deviation in the case of bromobenzene system is still larger than the one obtained using conventional PIMD. Moreover, the large SD for ${ }^{81} \mathrm{Br}$ isotope of $\mathrm{BB}$ suggests that energy of the given atom wildly variates, and most likely in a large unphysical way as mentioned in previous studies with halogen atoms ${ }^{76}$. Given behaviour might help to explain the SCC convergence problems along with large SD.

In contrast to conventional PIMD, carbon position specific isotope effects for the ETH system are distinguishable from each other, a clear effect of treating the system quantum mechanically. Even though isotope effect was not quantitatively reproduced, partially unbiased semiempirical DFTB still captures the difference between position $\mathrm{C}_{1}$ and $\mathrm{C}_{2}$, and provides both normal effects (negative values). However, the predicted trend is not in accord with the experimental pattern: $\mathrm{C}_{1}$ position exhibits larger whereas $\mathrm{C}_{2}$ position smaller effect. Experimental values of $\mathrm{Br}$ VPIEs on bromobenzene evaporation are reproduced, albeit possess a large SD.

Due to the aforementioned convergence complications the $S C C$-DFTB Suzuki-Chin PIMD simulations were carried out only for ethanol, specifically for the $\mathrm{C}_{1}$ atom. For a set of 8 imaginary slices predicted VPIE is equal to $-0.20 \pm 0.36 \varepsilon \%$. This result does not only offer a smaller SD with the same number imaginary slices as compared to $S C C$-DFTB PIMD, but also VPIE is larger and closer to the measured value. It should be mentioned that a time step of 0.4 fs was used in $S C C$-DFTB Suzuki-Chin
PIMD since larger time steps introduced SCC convergence issues.

Although SCC-DFTB offers a promising alternative to any force field method its performance with respect to prediction of isotope effects of very small magnitude like VPIEs is questionable and requires improvement. Very recent study from our laboratory also on VPIEs from ethanol but under distillation conditions clearly indicates necessity of using quantum method along with proper solvation model providing an adequate first solvation shell description (using of at least cluster model of a size of several ethanol molecules) 82 .

\section{Interaction energies and magnitudes of predicted VPIEs}

Differences in predicted VPIEs between OPLS and $S C C$-DFTB are substantial. They arise from the level of theory at which compounds are treated, therefore in order to explore how this different theoretical treatment influences the interactions between molecules and thus indirectly predicted isotope effects, we used the symmetryadapted perturbation theory (SAPT) ${ }^{83}$. This energy decomposition analysis permits to obtain a better insight into fundamental characteristics of compounds intermolecular interactions. With this in mind we studied the interaction energy $\left(E_{\text {int }}\right)$ and its direct decomposition in into four physically meaningful components i.e. electrostatic, exchange, induction, and dispersion.

In order to ensure a rigorous calculation of interaction energies, a highly accurate methodology was used, namely, high-order SAPT with correlation-consistent augmented triple Dunning basis set ${ }^{[4 \mid 85}$, supermolecular MP2 interaction energy ( $\delta \mathrm{MP} 2)$ and coupled-cluster doubles (CCD) for dispersion 1867 , (SAPT2+(CCD) $\delta \mathrm{MP} 2 / \mathrm{aTZ})$. $E_{\text {int }}$ were calculated for clusters (Fig. 4 and Fig. S2 in Supplementary Material) of four molecules extracted from the MD simulations using OPLS and $S C C$-DFTB in the

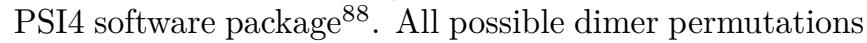
within each cluster were considered.

We compared $E_{\text {int }}$ obtained for both OPLS and $S C C$ DFTB geometries in Fig. 5. Results indicate that intermolecular interactions are slightly stronger and evenly distributed in the case of OPLS force field. Moreover, it is important to realise the evident visual difference in the structures between $S C C-D F T B$ and OPLS for Dibromomethane. OPLS structrure is compacter, showing multiple interactions between $\mathrm{H}$ and $\mathrm{Br}$ atoms of different molecules. In the case of $S C C-D F T B$, intermolecular interactions are biased towards $\mathrm{Br}$ atoms, thus showing a possible overestimation of the interactions in this particular atom $(\mathrm{Br})$. Nevertheless, it is worth noticing that energy difference between the strongest interactions for the same compound at different level of theory do not surpass $1 \mathrm{kcal} \mathrm{mol}^{-1}$. This indicates that both OPLS and $S C C$-DFTB provide a similar description of compounds, in terms of energy strictly speaking. 
TABLE IV. VPIEs $(\varepsilon \%)$ predicted on evaporation for bromobenzene, and ethanol using SCC-DFTB PIMD.

\begin{tabular}{lcccc}
\hline & & \multicolumn{2}{c}{ Time slices } & \multicolumn{1}{c}{16} \\
\cline { 3 - 4 } Compound & isotope & 8 & $-0.81 \pm 1.57$ & $-0.9 \pm 0.2^{\mathrm{a}}$ \\
\hline BB & ${ }^{81} \mathrm{Br}$ & $-1.06 \pm 1.73$ & $-0.15 \pm 0.35$ \\
ETH & ${ }^{13} \mathrm{C}_{1}$ & $-0.14 \pm 0.40$ & $-0.23 \pm 0.32$ & $-6.11^{\mathrm{b}}$ \\
ETH & ${ }^{13} \mathrm{C}_{2}$ & $-0.22 \pm 0.37$ & $-2 . \mathrm{b}^{\mathrm{b}}$ \\
\hline
\end{tabular}

aRef. 48.

bRef. 24 by passive evaporation.

SCC-DFTB
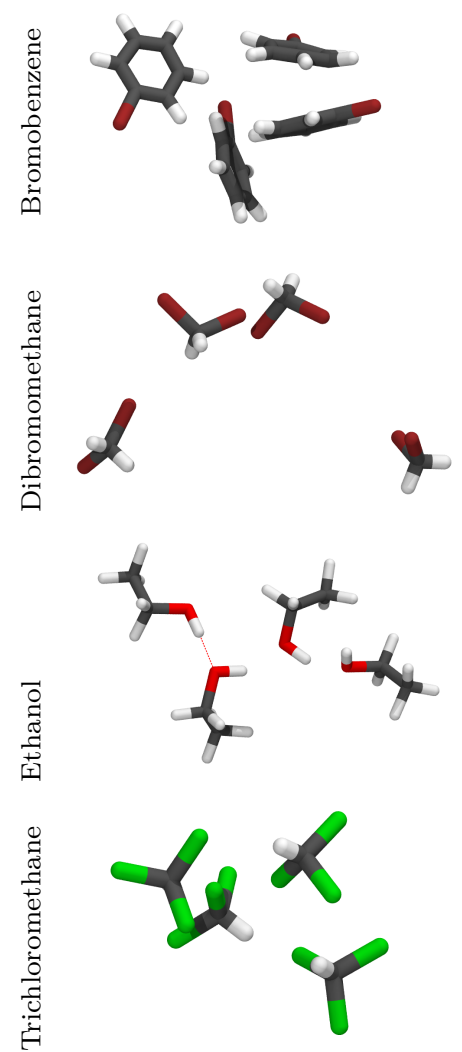

FIG. 4. Cluster models of all compounds clusters utilised for the energy decomposition analysis.

Comparison of $E_{\text {int }}$ components i.e. electrostatics, exchange, induction and dispersion cannot be performed directly as only in the case of $S C C$-DFTB aforementioned forces are being properly evaluated. Given that OPLS is a force field, it does not evaluate explicitly for example dispersion or exchange, instead it does it implicitly via prefactors and parameters. Thus, although for the OPLS interaction energy might be approximated correctly, its meaningful physical components are not. For instance ethanol clusters (see Fig. S2 in Supplementary Material) are interacting via hydrogen bonding (three bonds), average interaction energy at high level of theory for ethano 89 dimer is $6.5 \mathrm{kcal} \mathrm{mol}^{-1}$. However, we found that in the case of OPLS, only one interacting dimer possesses a close to the high level of theory value of interaction en-
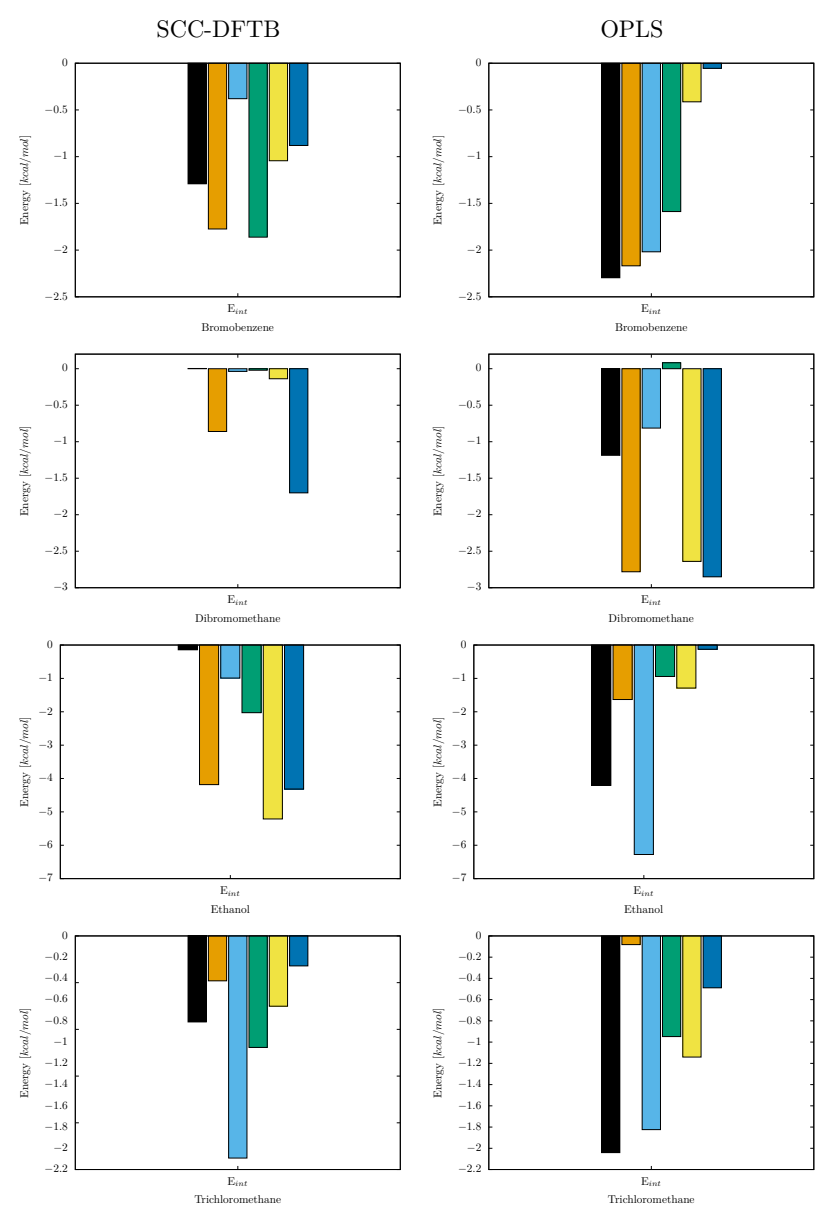

FIG. 5. Interaction energies for all permutations (shown as color bars) within each four molecule cluster obtained from geometries obtained at the $S C C$-DFTB (left) and OPLS (right) levels of theory.

ergy and the next closer at around $4 \mathrm{kcal} \mathrm{mol}^{-1}$ (black bar in Fig 5), whereas $S C C-D F T B$ indeed gives three dimers with relatively strong $E_{\text {int }}$.

\section{CONCLUSIONS}

In this work, we have investigated the performance of several methodologies, some recently developed, to predict VPIE on evaporation from pure ethanol, chloroform, 
bromobenzene, and dibromomethane solvents using the path integral principle. To reliably calculate VPIE, a decently accurate reproduction of the potential energy surface of the system, is mandatory.

For this precise reason, although a trusty potential energy surface is available even at the force field level, and carbon isotope effects on ethanol vaporisation can be qualitatively reproduced the differences in position specific isotope effects could not be captured. In order to achieve it, at least semi-empirical like $S C C-D F T B$ calculations should be performed. Nevertheless, opting for an $S C C-D F T B$ method consequently means that quantisation of even small systems is going to be demanding and computationally more expensive. VPIE obtained for ethanol and bromobenzene using the DFTB3 method, generally speaking, were in qualitatively good agreement with the experimental data. Unfortunately for the other studied systems (DBM and TCM), it was unachievable to calculate VPIE, owing to serious issues with the SCC convergence.

One of the characteristics that the challenging systems share is the presence of at least two halogen atoms in their composition, which based on our study, seems to be the source of the SCC convergence problems. A possible approach to solve this problem is utilising the recently developed scheme DFTB3/3OB(XB)-D3H5 5 , which includes corrections for non-covalent interactions such as dispersion, hydrogen bonds, and halogen bonds. Nonetheless, given scheme is still under development and not found yet in the release of DFTB+ stable version.

$S C C$-DFTB Suzuki-Chin path integral molecular dynamics demonstrated to be a more efficient way to calculate isotope effects. It outperformed the classical $S C C$ DFTB PIMD by utilising a smaller number of imaginary time slices, it did not only produce a smaller standard deviation, but also decreased by almost half the computational cost of the simulations.

Even though $S C C$-DFTB Suzuki-Chin PIMD in general appears to be a better option than SCC-DFTB PIMD, we found that the former approach required slightly smaller time steps during the ensemble than the latter one, at least for the studied systems in order to avoid convergence issues.

All things considered, it is important to mention that to the best of our knowledge, apart from water and halides ${ }^{91}$, there are no examples of isotope effects calculation for pure phase solvents using PIMD, or SCC-DFTB PIMD with the same purpose. Hence, this study serves as a robust test for the methods used, and provides evidence about the current limits of force fields potentials and $S C C-D F T B$ in reproducing parameters rising from mainly non-covalent interactions of different kind: dispersion, hydrogen bonds, halogen bonds, etc.

\section{ACKNOWLEDGMENTS}

The authors thank Venkat Kapil and Prof. Dorota Swiatla-Wojcik for helpful discussions, and are grateful to Dr. Szilvia Pothoczki for sharing her experimental data on trichloromethane as well as comments on our findings. This work was supported by the National Science Centre in Poland (Sonata BIS grant UMO2014/14/E/ST4/00041) and in part by PLGrid Infrastructure (Poland).

${ }^{1}$ F. A. Lindemann, The London, Edinburgh, and Dublin Philosophical Magazine and Journal of Science 38, 173 (1919)

${ }^{2}$ F. A. Lindemann and F. W. Aston, The London, Edinburgh, and Dublin Philosophical Magazine and Journal of Science 37, 523 (1919)

${ }^{3}$ J. Bigeleisen and M. G. Mayer, The Journal of Chemical Physics 15, 261 (1947)

${ }^{4}$ J. Bigeleisen, The Journal of Chemical Physics 17, 675 (1949)

${ }^{5}$ M. J. Stern, W. A. Van Hook, and M. Wolfsberg, The Journal of Chemical Physics 39, 3179 (1963)

${ }^{0}$ J. Bigeleisen, S. V. Ribnikar, and W. A. V. Hook, The Journal of Chemical Physics 38, 489 (1963)

'J. Bigeleisen, M. J. Stern, and W. A. Van Hook, The Journal of Chemical Physics 38, 497 (1963)

${ }^{\gamma}$ V. I. Ustinov and M. V. Petropavlov, Russ. J. Phys. Chem. (Engl. Transl.) 47, 1697 (1973).

${ }^{9}$ H. Craig and L. I. Gordon, in Stable Isotopes in Oceanographic Studies and Paleotemperatures edited by E. Tongiogi (Consiglio nazionale delle richerche, Laboratorio de geologia nucleare, Spoleto, Italy, 1965) pp. 9-130.

${ }^{10} \mathrm{R}$. Mills, Nature 256, 638 (1975)

${ }^{11} \mathrm{H}$. Wolff and A. Höpfner, Berichte der Bunsengesellschaft für physikalische Chemie 71, 730 (1967), https://onlinelibrary.wiley.com/doi/pdf/10.1002/bbpc.19670710717

${ }^{12}$ J. Horita, K. Rozanski, and S. Cohen, Isotopes in Environmental and Health Studies 44, 23 (2008)

${ }^{15}$ G. D. Farquhar, J. R. Ehleringer, and K. T. Hubick, Annual Review of Plant Physiology and Plant Molecular Biology 40, 503 (1989), https://doi.org/10.1146/annurev.pp.40.060189.002443.

${ }^{14}$ M. M. Barbour, J. S. Roden, G. D. Farquhar, and J. R. Ehleringer, Oecologia 138, 426 (2004)

${ }^{15}$ M. M. Barbour, Functional Plant Biology 34, 83 (2007)

${ }^{16}$ S. Jeannottat and D. Hunkeler, Environmental Science \& Technology 46, 3169 (2012)

${ }^{1 /}$ P. G. K. van Groos, B. K. Esser, R. W. Williams, and J. R. Hunt, Environmental Science \& Technology 48, 227 (2013).

${ }^{18}$ J. Bigeleisen, The Journal of Chemical Physics 34, 1485 (1961)

${ }^{19}$ J. S. Pollin and T. Ishida, The Journal of Chemical Physics 66, $4442(1977)$

${ }^{20}$ T. Oi, J. Shulman, A. Popowicz, and T. Ishida, The Journal of Physical Chemistry 87, 3153 (1983)

${ }^{21}$ J. Szydłowski, Journal of Molecular Structure 321, 101 (1994)

${ }^{22}$ M. Wolfsberg, W. A. Hook, P. Paneth, and L. P. N. Rebelo, Isotope Effects (Springer Netherlands, Dordrecht, 2009).

${ }^{23}$ T. Kuder, P. Philp, and J. Allen, Environmental Science \& Technology 43, 1763 (2009), pMID: 19368169, https://doi.org/10.1021/es802834p

${ }^{24}$ M. Julien, P. Nun, R. J. Robins, G. S. Remaud, J. Parinet, and P. Höhener, Environmental Science \& Technology 49, 12782 (2015)

${ }^{25}$ M. Julien, P. Hoöhener, R. J. Robins, J. Parinet, and G. S. Remaud, The Journal of Physical Chemistry B 121, 5810 (2017)

${ }^{26}$ G. J. Martin and M. L. Martin, Tetrahedron Letters 22, 3525 (1981)

${ }^{2}$ A. Popowicz, T. Oi, J. Shulman, and T. Ishida, The Journal of Chemical Physics 76, 3732 (1982) 
${ }^{28}$ A. Kanungo, T. Oi, A. Popowicz, and T. Ishida, The Journal of Physical Chemistry 91, 4198 (1987)

${ }^{29}$ A. Horst, G. Lacrampe-Couloume, and B. S. Lollar, Analytical Chemistry 88, 12066 (2016)

${ }^{30} \mathrm{H}$. Mehrer, Diffusion in Solids (Springer Berlin Heidelberg, Berlin, Heidelberg, 2007).

${ }^{31}$ D. Bouchard, P. Höhener, and D. Hunkeler, Environmental Science \& Technology 42, 7801 (2008), pMID: 19031863, https://doi.org/10.1021/es800391s

${ }^{32}$ F.-D. Kopinke and A. Georgi, Analytical Chemistry 89, 10637 (2017)

${ }^{3}$ M. Julien, J. Parinet, P. Nun, K. Bayle, P. Höhener, R. J. Robins, and G. S. Remaud, Environmental Pollution 205, 299 (2015)

${ }^{34}$ S. Yanase and T. Oi, Chemical Physics Letters 440, 19 (2007)

${ }^{35}$ T. Oi, R. Mitome, and S. Yanase, Zeitschrift für Naturforschung

A 72, 061701 (2017)

${ }^{36}$ R. P. Feynman and A. R. Hibbs, English Quantum Mechanics and

Path Integrals: Emended Edition, 1st ed. (McGraw-Hill Book Company, New York, St. Louis, San Francisco, Toronto, London, Sydney, 1965).

${ }^{37}$ R. P. Feynman, Statistical Mechanics: A Set Of Lectures (Frontiers in Physics) (W. A. Benjamin, 1972).

${ }^{38}$ B. J. Berne and D. Thirumalai, Annual Review of Physical Chemistry 37, 401 (1986)

${ }^{39}$ T. E. Markland and B. J. Berne, Proceedings of the $\mathrm{Na}-$ tional Academy of Sciences 109, 7988 (2012), arXiv:1307.7684v1 [physics.chem-ph]

${ }^{40}$ M. Ceriotti and T. E. Markland, The Journal of Chemical Physics 138, 014112 (2013) arXiv:1211.1475 [cond-mat.stat-mech]

${ }^{41}$ O. Marsalek, P.-Y. Chen, R. Dupuis, M. Benoit, M. Méheut, Z. Bačić, and M. E. Tuckerman, Journal of Chemical Theory and Computation 10, 1440 (2014), arXiv:1801.05888 [physics.chem$\mathrm{ph}]$

${ }^{42}$ B. Cheng and M. Ceriotti, The Journal of Chemical Physics 141, 244112 (2014) arXiv:1412.1308 [physics.chem-ph].

${ }^{45}$ M. Ceriotti, W. Fang, P. G. Kusalik, R. H. McKenzie, A. Michaelides, M. A. Morales, and T. E. Markland, Chemical Reviews 116, 7529 (2016)

${ }^{44}$ D. Chandler and P. G. Wolynes, Journal of Chemical Physics 74, 4078 (1981)

${ }^{45}$ J. Vaníček, W. H. Miller, J. F. Castillo, and F. J. Aoiz, The Journal of Chemical Physics 123, 054108 (2005)

${ }^{40}$ J. Vaníček and W. H. Miller, The Journal of Chemical Physics 127, 114309 (2007)

${ }^{41}$ P. S. of London. and H. v. Helmholtz, Physical memoirs selected and translated from foreign sources, under the direction of the Physical society of London. Vol. 1 (Taylor and Francis, London, 1891).

${ }^{48}$ L. Vasquez, M. Rostkowski, F. Gelman, and A. Dybala-Defratyka, The Journal of Physical Chemistry B 122, 7353 (2018)

${ }^{49}$ S. Plimpton, Journal of Computational Physics 117, 1 (1995)

${ }^{50}$ W. L. Jorgensen, D. S. Maxwell, and J. Tirado-Rives, Journal of

the American Chemical Society 118, 11225 (1996)

${ }^{51}$ W. L. Jorgensen and J. Tirado-Rives, Journal of the American Chemical Society 110, 1657 (1988)

${ }^{52}$ L. S. Dodda, I. CabezaădeăVaca, J. Tirado-Rives, and W. L. Jorgensen, Nucleic Acids Research 45, W331 (2017)

${ }^{53}$ G. J. Martyna, M. L. Klein, and M. Tuckerman, The Journal of Chemical Physics 97, 2635 (1992)

${ }^{54}$ W. Shinoda, M. Shiga, and M. Mikami, Physical Review B 69, 134103 (2004)

${ }^{55}$ M. Tuckerman, B. J. Berne, and G. J. Martyna, Journal of Chemical Physics 97, 1990 (1992)

${ }^{56}$ V. Kapil, M. Rossi, O. Marsalek, R. Petraglia, Y. Litman, T. Spura, B. Cheng, A. Cuzzocrea, R. H. Meißner, D. M. Wilkins, B. A. Helfrecht, P. Juda, S. P. Bienvenue, W. Fang, J. Kessler, I. Poltavsky, S. Vandenbrande, J. Wieme, C. Corminboeuf, T. D. Kühne, D. E. Manolopoulos, T. E. Markland, J. O. Richardson, A. Tkatchenko, G. A. Tribello, V. V. Speybroeck, and M. Ceriotti, Computer Physics Communications 236, 214 (2019)
$1808.03824 \mathrm{v} 2$

${ }^{57}$ M. Ceriotti, M. Parrinello, T. E. Markland, and D. E. Manolopoulos, The Journal of Chemical Physics 133, 124104 (2010)

${ }^{58}$ L. Pereyaslavets, I. Kurnikov, G. Kamath, O. Butin, A. Illarionov, I. Leontyev, M. Olevanov, M. Levitt, R. D. Kornberg, and B. Fain, Proceedings of the National Academy of Sciences 115, 8878 (2018)

${ }^{5}$ W. M. Haynes, EnglishHaynes, W. M., ed. 2014, CRC Handbook of Chemistry and Physics, 95th edn. (Boca Raton, FL: CRC Press), ISBN 9781482208689. 95th ed. (Oakville: CRC Press, 2014) proQuest Ebook Central ATO Loan.

${ }^{60}$ J. A. Dean, J. G. Speight, and N. A. Lange, EnglishLange's

Handbook of Chemistry, Seventeenth Edition seventeenth edition ed. (McGraw-Hill Education, 2017) previous edition: 2005.

${ }^{61}$ ChemicalBook, Dibromomethane chemical properties (2019).

${ }^{62} \mathrm{H}$. Bertagnolli, T. Engelhardt, and B. Lengeler, Berichte der Bunsengesellschaft für physikalische Chemie 91, 1287 (1987)

${ }^{63}$ S. Pothoczki, L. Temleitner, S. Kohara, P. Jóvári, and L. Pusztai, Journal of Physics: Condensed Matter 22, 404211 (2010).

${ }^{64}$ C.-C. Yin, A. H.-T. Li, and S. D. Chao, The Journal of Chemical Physics 139, 194501 (2013)

${ }^{5}$ A. Vrhovšek, O. Gereben, S. Pothoczki, M. Tomšič, A. Jamnik, S. Kohara, and L. Pusztai, Journal of Physics: Condensed Matter 22, $404214(2010)$

${ }^{66}$ A. Vrhovšek, O. Gereben, A. Jamnik, and L. Pusztai, The Journal of Physical Chemistry B 115, 13473 (2011)

${ }^{67}$ O. Gereben and L. Pusztai, The Journal of Physical Chemistry B 119, 3070 (2015)

${ }^{68}$ M. Liu, K. N. Youmans, and J. Gao, Molecules 23, 2644 (2018)

${ }^{69}$ D. Porezag, T. Frauenheim, T. Köhler, G. Seifert, and R. Kaschner, Physical Review B 51, 12947 (1995)

${ }^{70}$ S. A. Chin, Physics Letters A 226, 344 (1997)

${ }^{71}$ S. Jang, S. Jang, and G. A. Voth, Journal of Chemical Physics 115, 7832 (2001)

${ }^{72}$ M. Suzuki, Physics Letters A 201, 425 (1995).

${ }^{73}$ V. Kapil, J. Behler, and M. Ceriotti, Journal of Chemical Physics 145, 234103 (2016)

${ }^{14}$ B. Aradi, B. Hourahine, and T. Frauenheim, The Journal of Physical Chemistry A 111, 5678 (2007) https://doi.org/10.1021/jp070186p

${ }^{75} \mathrm{M}$. Gaus, A. Goez, and M. Elstner, Journal of Chemical Theory and Computation $\mathbf{9 ,} 338$ (2012) https://doi.org/10.1021/ct300849w

${ }^{76}$ M. Kubillus, T. Kubař, M. Gaus, J. Řezáč, and M. Elstner, Journal of Chemical Theory and Computation 11, 332 (2015) https://doi.org/10.1021/ct5009137

${ }^{77} \mathrm{~S}$. Grimme, J. Antony, S. Ehrlich, and H. Krieg, Journal of Chemical Physics 132, $154104 \quad$ (2010) https://doi.org/10.1063/1.3382344

${ }^{78}$ J. Rezáč and P. Hobza, Journal of Chemical Theory and Computation 8, 141 (2011), https://doi.org/10.1021/ct200751e

${ }^{{ }^{y}} \mathrm{~V}$. M. Miriyala and J. Rezáč, Journal of Computational Chemistry 38, 688 (2017), https://www.onlinelibrary.wiley.com/doi/pdf/10.1002/jcc.24725.

${ }^{80}$ J. Rezáč, Journal of Chemical Theory and Computation 13, 4804 (2017), https://doi.org/10.1021/acs.jctc.7b00629

${ }^{81}$ M. Ceriotti, G. Bussi, and M. Parrinello, Physical Review Letters 103, $030603(2009)$

${ }^{\mathrm{\gamma}} \mathrm{K}$. Klajman, A. Dybala-Defratyka, and P. Paneth, ACS Omega 10.1021/acsomega.0c02446 (2020).

${ }^{83}$ B. Jeziorski, R. Moszynski, and K. Szalewicz, Chemical Reviews 94, 1887 (1994)

${ }^{84}$ T. H. Dunning, The Journal of Chemical Physics 90, 1007 (1989)

${ }^{85}$ R. A. Kendall, T. H. Dunning, and R. J. Harrison, The Journal of Chemical Physics 96, 6796 (1992)

${ }^{80}$ R. M. Parrish, E. G. Hohenstein, and C. D. Sherrill, The Journal of Chemical Physics 139, 174102 (2013)

${ }^{87}$ T. M. Parker, L. A. Burns, R. M. Parrish, A. G. Ryno, and C. D. Sherrill, The Journal of Chemical Physics 140, 094106 (2014)

${ }^{88}$ R. M. Parrish, L. A. Burns, D. G. A. Smith, A. C. Simmonett, 
A. E. DePrince, E. G. Hohenstein, U. Bozkaya, A. Y. Sokolov, R. D. Remigio, R. M. Richard, J. F. Gonthier, A. M. James, H. R. McAlexander, A. Kumar, M. Saitow, X. Wang, B. P. Pritchard, P. Verma, H. F. Schaefer, K. Patkowski, R. A. King, E. F. Valeev, F. A. Evangelista, J. M. Turney, T. D. Crawford, and C. D. Sherrill, Journal of Chemical Theory and Computation 13, 3185 (2017)
${ }^{89}$ A. Vargas-Caamal, F. Ortiz-Chi, D. Moreno, A. Restrepo, G. Merino, and J. L. Cabellos, Theoretical Chemistry Accounts 134, 10.1007/s00214-015-1615-9 (2015).

${ }^{90}$ J. Řezáč, Journal of Computational Chemistry 40, 1633 (2019)

${ }^{91}$ P. E. Videla, P. J. Rossky, and D. Laria, The Journal of Chemical Physics 148, 102306 (2018) 